\title{
Photodegradation of selected phthalates on mural painting surfaces under UV light irradiation
}

\author{
Salvatore Barreca, Roberta Indelicato, Santino Orecchio *, Andrea Pace \\ Dipartimento di Scienze e Tecnologie Biologiche, Chimiche e Farmaceutiche, Università di Palermo, Viale delle Scienze, I-90128 Palermo, Italy
}

\section{A R T I C L E I N F O}

\section{Article history:}

Received 20 December 2013

Accepted 3 January 2014

Available online 10 January 2014

Keywords:

Mural paintings

Phthalates

GC-MS

Restore

Construction

\begin{abstract}
A B S T R A C T
The esters of phthalic acid are considered as hazardous pollutants due to their mutagenicity, carcinogenicity and are also classified as endocrine disruptor chemicals. Several compounds of this class of substances for decades, and probably even now, were used as softeners in water-based synthetic paintings. Surfaces and structures, such as house walls painted with phthalates based paintings, can be a concern to construction workers engaged in demolition, restore and paint removal activities if they are not protected from hazardous dust inhalation. In this paper we report the results of an investigation about phthalate ester degradation by direct UV irradiation at $254 \mathrm{~nm}$. The results of kinetic parameters for PAE photodegradation are reported and it shows that $k$ values for the single PAEs ranged from 0.221 to $0.737 \mathrm{~h}^{-1}$.

Moreover, the results indicate that photolysis is a successful way to remediate PEAs contaminated on mural painting.
\end{abstract}

(C) 2014 Elsevier B.V. All rights reserved.

\section{Introduction}

The esters of phthalic acid $\left(\mathrm{C}_{6} \mathrm{H}_{4}(\mathrm{COOH})_{2}\right)$, generally called phthalates (PAEs), are a group of contaminants that are ubiquitous in the environment. These chemicals, generally, are colorless and odorless liquids having high boiling points, low volatility, insolubility in water and predominantly fat solubility. Not including the dimethyl phthalate (DMP) that belongs to the group of VOCs, PAEs are classified as semivolatile organic compounds. There are no natural sources of PAEs, therefore all phthalates found in environmental matrices can be attributed to man activities [1].

In industrial countries, anthropogenic activities are the principal sources of PAEs in environmental and building matrices [2,3]. Several PAEs are considered as hazardous pollutants due to their mutagenicity, carcinogenicity and are also classified as Endocrine Disruptor Chemicals (EDCs) [4-6].

The main processes which successfully remove and eliminate PAEs from the environmental matrices include: microbiological transformation and degradation, volatilization, photo-oxidation, chemical oxidation, sorption and biological uptake [7,8]. However, some of the PAEs are recalcitrants, and biological processes are not always effective in removing them. In this context, photodegradation is an important transformation pathway for most PAEs in the environment.

The compounds considered in this paper are the most used in industrial processes which are expected to be present in indoor environments: dimethyl phthalate (DMP), diethyl phthalate (DEP), di-n-butyl phthalate (DnBP), benzyl butyl phthalate (BBzP), bis(2-ethylhexyl)

\footnotetext{
* Corresponding author. Tel.: + 3909123897968.

E-mail address: santino.orecchio@unipa.it (S. Orecchio).
}

phthalate (DEHP), and di-n-octyl phthalate (DnOP). Compounds with higher molecular weights, such as bis(2-ethylhexyl) phthalate (DEHP), are largely used as additives and plasticizers, while those with lower molecular weights (diethyl, di-n-butyl and dimethyl phthalate) are components of industrial solvents, adhesive, wax, ink, pharmaceutical products, insecticide materials, and cosmetic [9,10]. DEHP was found in medical disposal devices and in a number of medicine coatings. Some compounds are contained in cleaning solutions for contact lenses [11] and in food packaging films [12].

Phthalates are not chemically but only physically bound to the polymer chains; hence, they may be leached into the environment and are ubiquitously found in air, water, soils, and sediments [13-17]. People exposure to PAEs may arise from toys, child-care articles, building materials, home furnishing, car interiors, clothing, medical devices, and foodcontact materials. PAEs are emitted into the atmosphere as particulates and gases [18]. Indoor environments increase the lifetime of pollutants adsorbed to the dust by minimizing or eliminating the natural decomposition processes catalyzed by natural light and rain [19].

Moreover, some phthalate esters for decades, and probably even now, were used as softeners in water-based synthetic paintings [2]. Di-n-butyl phthalate (DBP) and Bis(2-ethylhexyl) phthalate (DEHP) are the most employed. Softeners are released from the painted surface for a long time after they were applied [5]. Surfaces and structures, such as house walls painted with phthalates based paintings, can be a concern to construction workers engaged in demolition, restore and paint removal activities if they are not protected from hazardous dust inhalation.

In the last years, scientific community has focused his interest in processes that may be applied to degrade hazardous substances [20], minimizing the risk for worker exposures. Given that UV-irradiation at 
$254 \mathrm{~nm}$ is used for wastewater and soil treatments, it is of interest to know whether this process can also be employed for phthalate degradation onto mural painting surfaces because construction work is one of the most important activities in Europe, and probably worldwide, with respect to its economic, technological, and environmental impact. Dusts containing PAEs and other hazardous substances are generated in these processes, and during transporting, storing and handling construction materials [21]. This may also be verified when certain construction processes are undertaken. Extended work times on building matrices especially in the indoor environment increase possibility of exposure to contaminants by 1000 -fold compared to outdoor exposures [22]. Levels of phthalates in indoor air and dust are often higher than outdoor levels.

Currently, there is limited information concerning the UV photolysis of phthalates and in this paper we report the results of an investigation of the degradation by UV irradiation at a wavelength of $254 \mathrm{~nm}$. The described approach includes the choice of appropriate irradiation conditions in order to obtain the best results.

GC/MS technique was used to quantify PAEs in samples, taken from wall surface of building located in Palermo, before and after the irradiation.

\section{Experimental}

\subsection{Study area}

The mural painting samples taken in consideration in this study were sampled in eight buildings located in Palermo area (Italy) (Table 1) [3]. Palermo is a densely populated city (about 850,000 inhabitants) characterized by conspicuous air pollution [3,23-29]. Palermo is a typically European town in building style and is generally built in stone (tuff), clay bricks and concrete.

In order to determine a possible pattern in usage of PAEs in the Palermo area, sampling was conducted in different parts of the town, in different types of buildings of different ages (Table 1). The samples were taken, during 2012 from the surface of the indoor walls and were collected by scraping off the layers or removing fragments. From each surface, a total of 5 samples were collected. About $25 \mathrm{~g}$ of the material was placed in glass containers. The samples were refrigerated $\left(4{ }^{\circ} \mathrm{C}\right)$, avoiding the exposure to light, and taken to the laboratory where they were frozen $\left(-20^{\circ} \mathrm{C}\right)$ until the photodegradation was performed. About $2 \mathrm{~g}$ of homogenized samples of mural painting was dried at $105{ }^{\circ} \mathrm{C}$ for overnight. The water content was determined by weight loss and was utilized to correlate all the results with dry weight. Before each analysis the samples were finely pulverized in a mortar.

\subsection{Photoirradiation}

The mural painting samples (100-200 mg) were transferred into a Pyrex Petri dishes and directly irradiated for $8 \mathrm{~h}$ at $\lambda=254 \mathrm{~nm}$ by using a Mercury lamp located at $10 \mathrm{~cm}$. In order to monitor the irradiation effect as a function of time, samples were collected at 0 (analyzed without irradiation) $0.5,1,2,4,6$ and 8 h during the irradiation. All

Table 1

Descriptive profile of sampling station.

\begin{tabular}{lll}
\hline $\mathrm{n}^{\circ}$ & Characteristic of station & Age of building \\
\hline Mur 1 & Stair of a building built around 1990. & 23 \\
Mur 2 & Bedroom of an apartment on the first floor & 25 \\
Mur 3 & Stair of the previous station & 25 \\
Mur 4 & Living room of house dating from 1950 to & 63 \\
& 1 floor renovated 13 years ago. & 15 \\
Mur 5 & Kitchen of an apartment on the top floor. & 43 \\
Mur 6 & Garage & 30 \\
Mur 7 & Kitchen & 5 \\
Mur 8 & Kitchen & \\
\hline
\end{tabular}

the Petri dishes were randomly placed on the shelves, and sampling was performed during the irradiation with the sampled mural paintings sacrificed.

\subsection{Chemical analyses}

Concentrations of the PAEs in irradiated and non-irradiated samples were obtained by the following method. An aliquot of mural painting samples were transferred into $50 \mathrm{~mL}$ glass tubes, and mixed with hexane (100 mg of sample: $4 \mathrm{~mL}$ of hexane). Before extraction, surrogate standard (di-n-hexyl-phthalate- $\mathrm{d}_{4}$ ) (Table 2 ) was added to all samples for the evaluation of the method performance (yields). Each sample was extracted for $20 \mathrm{~min}$ ( 3 times) in an ultrasonic bath, in which the water temperature was just lower than $30{ }^{\circ} \mathrm{C}$. The mixture was then centrifuged at $5000 \mathrm{rpm}$ for $5 \mathrm{~min}$ to separate the supernatant from the solid.

The extracts were cleaned using a short silica gel column containing anhydrous $\mathrm{Na}_{2} \mathrm{SO}_{4}$, earlier wetted with hexane, and concentrated in a rotary evaporator at $\mathrm{T}=50{ }^{\circ} \mathrm{C}$. The final volume was around $1 \mathrm{~mL}$. The last stage in the procedure involved drying the PAEs containing solution under a weak $\mathrm{N}_{2}$ flow at room temperature. The dry residue was dissolved in $1 \mathrm{~mL}$ of solution containing a perdeuterated internal standard (Bis(2-ethylhexyl)phthalate- $\mathrm{d}_{4}$ ) in cyclohexane. Analysis of solutions was carried out using a gas chromatograph coupled with a mass spectrometer (Shimadzu, mod. GCMS-QP2000) equipped with an SLB $5 \mathrm{~ms}$ ( $30 \mathrm{~m} 0.25$ i.d., $0.5 \mu \mathrm{m}$ ) (5\% diphenyl 95\% dimethyl siloxane) phase fused-silica capillary column from Supelco (Milano, Italy). The mass spectrometer was operated in electron impact (70 eV) and selected ion monitoring (SIM) mode. GC oven temperature for PAEs started at $60{ }^{\circ} \mathrm{C}$ (2 min.) and ramped to $325^{\circ} \mathrm{C}$ at $14.5^{\circ} \mathrm{C} / \mathrm{min}$ and maintained for 30 min.

Injection of $1 \mu \mathrm{L}$ sample was performed by the Shimadzu Auto Injector AOC-20I. The instrument was equipped with a split/splitless injector with glass wool injection port liner and splitless time of $2 \mathrm{~min}$. Total flow $21 \mathrm{~mL} / \mathrm{min}$ was used. The carrier gas was helium (flow rate $1.4 \mathrm{~mL} / \mathrm{min}$ ). In several cases, to measure phthalates, sample extracts were diluted prior to GC analyses.

\subsection{Quality assurance}

The detection limits (LOD) were estimated as $3 \sigma$ (three times the background noise) (IUPAC criterion) and like reported in previous

Table 2

List of the PAEs and abbreviations, the deuterated internal (underlined) and surrogate (italic) standards employed, the quantification and confirmation ions for SIM GC/MS mode.

\begin{tabular}{|c|c|c|c|}
\hline Compound & Abbr. & $\mathrm{N}^{\circ} \mathrm{CAS}$ & $\begin{array}{l}\text { Quantification and } \\
\text { confirmation } \\
\text { ions }(\mathrm{m} / \mathrm{z})\end{array}$ \\
\hline Dimethyl phthalate & DMP & $131-11-3$ & $163-194$ \\
\hline Diethyl phthalate & DEP & $84-66-2$ & $121-149$ \\
\hline Diisobutyl phthalate & $\mathrm{DiBP}$ & $84-69-5$ & $149-167$ \\
\hline Di-n-butyl phthalate & DBP & $84-74-2$ & $149-150$ \\
\hline Bis(2-methoxyethyl) phthalate & DMEP & $117-82-8$ & 59-149 \\
\hline Bis(4-methyl-2-pentyl) phthalate & BMPP & $146-50-9$ & $121-149$ \\
\hline Dinonyl phthalate & DNP & $86-76-4$ & $57-71$ \\
\hline Bis(2-ethoxyethyl) phthalate & DEEP & $605-54-9$ & $45-72$ \\
\hline Di pentyl phthalate & DPP & $131-18-0$ & $149-167$ \\
\hline Dihexyl phthalate & $\mathrm{DHxP}$ & $84-75-3$ & $76-104$ \\
\hline Bis(2-n-butoxyethyl) phthalate & DBEP & $117-83-9$ & $57-149$ \\
\hline Bis(2-ethylhexyl) phthalate & DEHP & $117-81-7$ & $113-149$ \\
\hline Di-ciclohexyl phthalate & DCHP & $84-61-7$ & $83-149$ \\
\hline Di-n-octil phthalate & DOP & $117-84-0$ & $149-150$ \\
\hline Benzyl butyl phthalate & BBP & $85-68-7$ & $91-149$ \\
\hline Diethyl phthalate- $\mathrm{d}_{4}$ & DEPd $_{4}$ & & 153 \\
\hline Bis(2-ethylhexyl)phthalate- $\mathrm{d}_{4}$ & $\mathrm{BEHP} \mathrm{d}_{4}$ & & 153 \\
\hline Di-n-hexyl-phthalate-d4 & $\mathrm{DHXPd}_{4}$ & & 153 \\
\hline
\end{tabular}




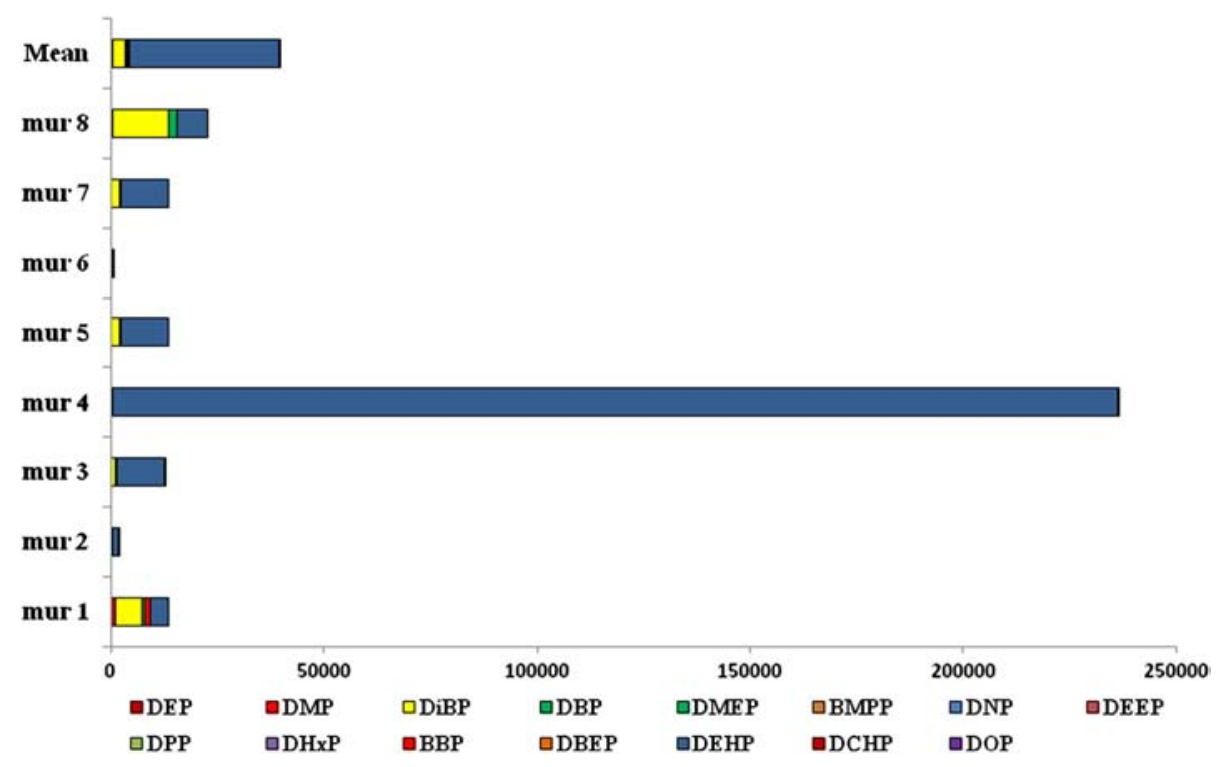

Fig. 1. Total and single PAEs (in $\mathrm{mg} / \mathrm{kg}$ ) (average of three analysis) concentrations in mural painting samples.

papers $[2,3,25,26,28]$ were similar for all analyzed compounds (less than $0.3 \mu \mathrm{g} / \mathrm{kg}$ for all compounds). Quantification limit (LOQ), (less than $1 \mu \mathrm{g} / \mathrm{kg}$ for all compounds) was estimated as $10 \sigma$ (ten times the background noise) (IUPAC criterion). LOD and LOQ were estimated in selected ion monitoring (SIM) mode [2,32 34, 36, 38]. Blank concentrations were not subtracted from sample concentrations because the levels in samples were higher than blank levels by several orders of magnitude.

The procedure was checked for recovery efficiencies as reported in a previous paper [3]. The average recoveries ranged from $85 \%$ to $99 \%$. The relative standard deviations on the PAE recovery were less than $10 \%$. Relative percent differences for triplicate samples were less than $14 \%$.

\section{Results and discussion}

The total initial concentrations of the investigated compounds, expressed as the sum of the concentrations, $\mathrm{PPAEs}$, in the analyzed mural painting, ranged from 0.8 to $236 \mathrm{mg} / \mathrm{kg}$ d.w. with an average of $39.4 \mathrm{mg} / \mathrm{kg}$ d.w. (Fig. 1) [3].

Among the 15 phthalates monitored only four (DEHP, DiBP, DBP and DEP) were detected in appreciable quantities [3]. BBP was significant only for one sample and at trace levels only in two mural paintings. In all tested samples, except $n^{\circ} 1$ and $n^{\circ} 8$, predominates the DEHP (from 30 to $100 \%$ of total phthalates) that is in the range from 0.6 to $236 \mathrm{mg} / \mathrm{kg}$ d.w. Sporadically, DiNP was used as a substitute to DEHP, however, in our case, its occurrence was not found.

The photodegradation loss can be obtained from the following equation:

$\mathrm{L}=\left(\mathrm{C}_{\mathrm{N}}-\mathrm{C}_{\mathrm{I}}\right) * 100 / \mathrm{C}_{0}$

where $\mathrm{L}=$ photodegradation loss at time $\mathrm{t}, \mathrm{C}_{0}=$ original concentration of single compound, $\mathrm{C}_{\mathrm{N}}=$ concentration of compound nonirradiated mural painting sample at time $\mathrm{t}, \mathrm{C}_{\mathrm{I}}=$ concentration of compound irradiated sample at time $t$.

The photodegradation losses of single PAE versus time on mural painting surfaces are shown in Fig. 2. The loss, as sum of investigated compounds, increased rapidly during the first $60 \mathrm{~min}$ of the experiment, with the degradation loss being about 57\%. As the photolysis reaction progressed with continuing irradiation, it was evident that the PAE degradation rate gradually reduced.

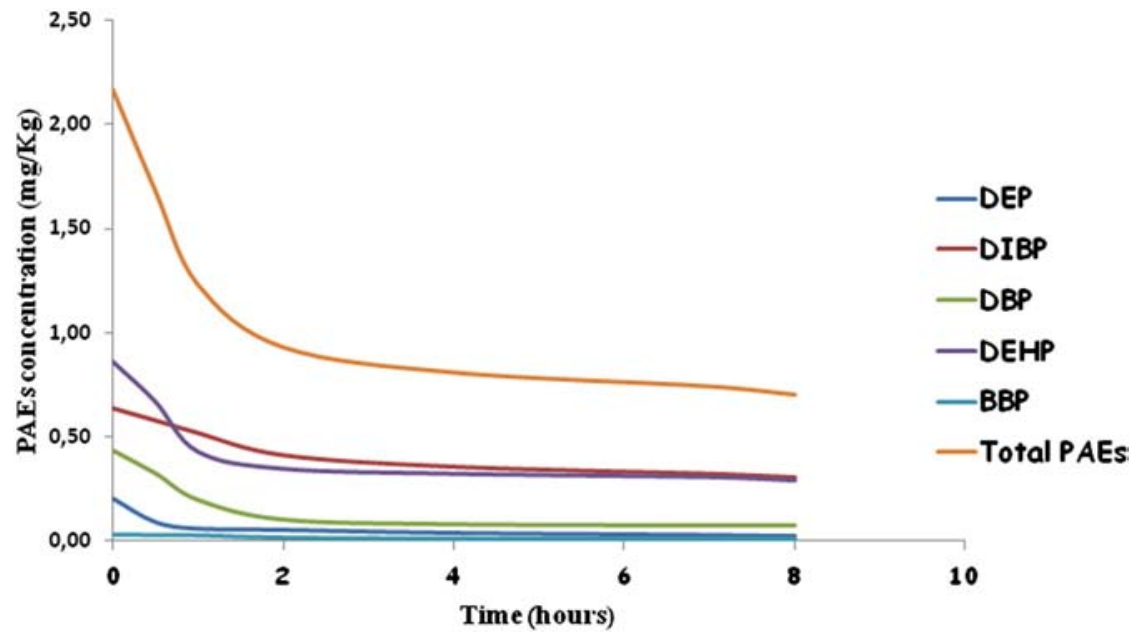

Fig. 2. Concentration of single PAE versus time on mural painting samples. 


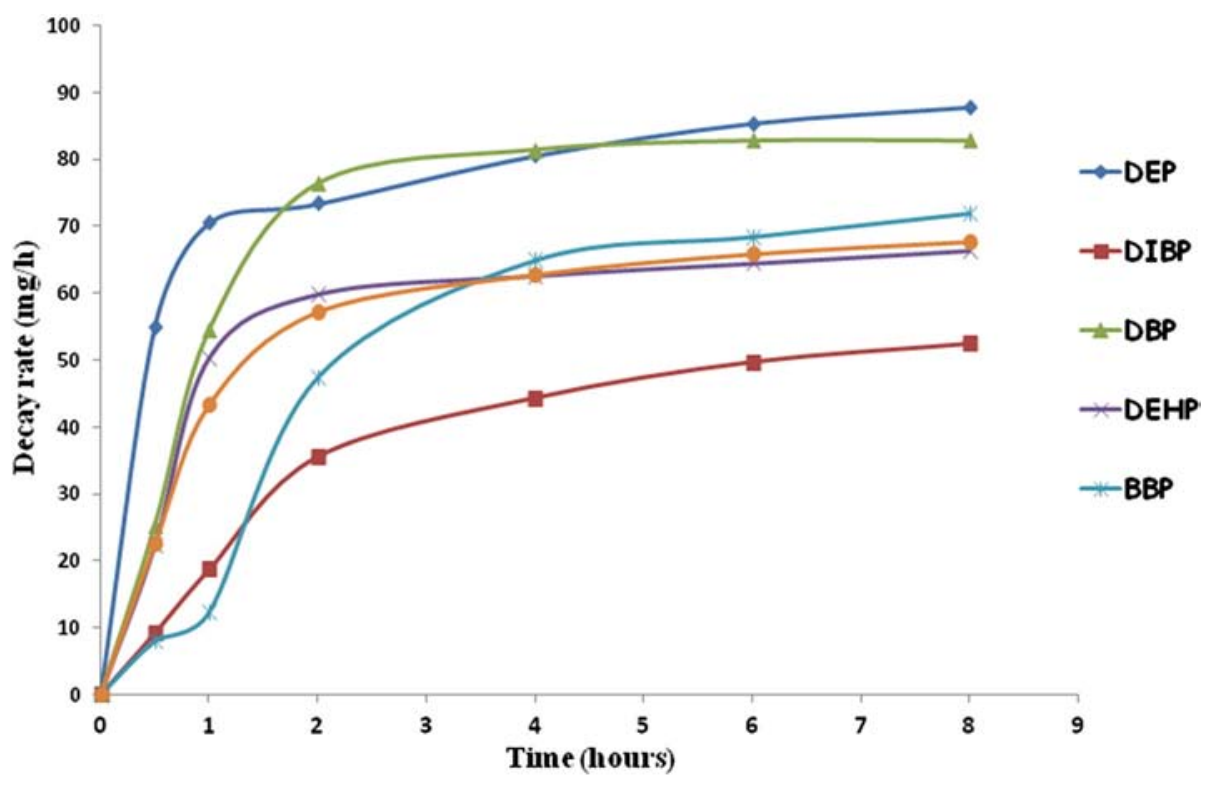

Fig. 3. Decay rate.

We can deduce that a significant removal (68\%) of total PAEs can be achieved by an irradiation period of $8 \mathrm{~h}$. The photolysis of the investigated compounds was then found to follow pseudo first kinetics order $\left(C_{T}=C_{0} e^{-k t}\right)$ initially; however, after a period of irradiation (60 min) the decay rate reduced, as shown in Fig. 3.

Considering the photodegradation loss of the compound found in higher concentrations in mural painting samples (DEHP) increased rapidly during the first $60 \mathrm{~min}$ of the treatment, with the degradation loss being about $50 \%$.

Our study showed that PAE photodegradation was fitted to the firstorder equation:

$\ln \mathrm{C}_{0} / \mathrm{C}=k \mathrm{t}$

where $\mathrm{C}_{0}$ and $\mathrm{C}$ are the PAE concentration at times zero and $\mathrm{t}$ respective$\mathrm{ly}$, and $\mathrm{k}$ is the rate constant. First-order degradation rate constants were determined by analysis. Half-lives $t_{1 / 2}$ were calculated using Eq. (3) which was derived from Eq. (2) by replacing $C$ with $C_{0} / 2$ :

$\mathrm{t}_{1 / 2}=\ln 2 / k=0.6931 / k$

The results of kinetic parameters for PAE photodegradation are listed in Table 3. It shows that $k$ values for the single PAEs ranged from 0.221 to $0.737 \mathrm{~h}^{-1}$.

The direct photolysis induced by UV at $254 \mathrm{~nm}$ should be one of the major reaction pathways responsible for the PAE decay.

In particular, such as reported in literature, the photodegradation of phthalate esters at $254 \mathrm{~nm}$ involves photo-induced decarboxylation, hydroxylation, dealkylation, and scission of $\mathrm{C}-\mathrm{O}, \mathrm{C}-\mathrm{C}$, and $\mathrm{O}-\mathrm{alkyl}$ bonds $[7,30,31]$.

Table 3

Kinetic parameters for single PAE photodegradation.

\begin{tabular}{llll}
\hline Compound & $k\left(\mathrm{~h}^{-1}\right)$ & $\mathrm{R}$ & $\mathrm{t}_{1 / 2}$ \\
\hline DEP & $\mathbf{0 . 6 3 1}$ & $\mathbf{0 . 8 7}$ & $\mathbf{1 . 1 5}$ \\
DIBP & 0.221 & $\mathbf{0 . 9 9}$ & $\mathbf{2 . 2 0}$ \\
DBP & 0.737 & $\mathbf{0 . 9 9}$ & $\mathbf{1 . 0 0}$ \\
DEHP & 0.466 & $\mathbf{0 . 9 5}$ & $\mathbf{1 . 4 6}$ \\
BBP & 0.323 & $\mathbf{0 . 9 5}$ & $\mathbf{1 . 8 2}$ \\
Total PAEs & 0.424 & $\mathbf{0 . 9 8}$ & $\mathbf{1 . 5 5}$ \\
\hline
\end{tabular}

\section{Conclusion}

The transformation and volatilization of PAEs in different aquatic systems with sunlight irradiation are irrelevant. The hydrolysis halflife of DBP is estimated at 22 years, and the atmospheric photooxidation half-life is reported to be between 0.6 and 6 days [32].

Photodegradation is a major degradation process which can clean up the mural paintings. According to our study, PAE photodegradation follows pseudo-first-order kinetics. The rate constant of photodegradation ranged from 0.22 to $0.74 \mathrm{~h}^{-1}$.

The results of the present study suggest that the use of UV irradiation before and during the execution of renovation or restoration may be wanted in an attempt to reduce exposure to PAEs. Those data should be helpful for structuring the phthalate exposure profile and recommending local exhaust systems and personal protection equipment for construction workers.

\section{Acknowledgment}

This study was made possible by the financial support of Palermo University that has funded the author (Grants ex 60\% 2007).

\section{References}

[1] M. Wormuth, M. Scheringer, M. Vollenweider, K. Hungerbuhler, What are the sources of exposure to eight frequently used phthalic acid esters in Europeans? Risk Anal. 26 (2006) 803-824.

[2] S. Orecchio, R. Indelicato, S. Barreca, Determination of selected phthalates by gas chromatography-mass spectrometry in mural paintings from Palermo (Italy), Microchemical Journal, http://dx.doi.org/10.1016/j.microc.2013.11015.

[3] S. Orecchio, R. Indelicato, S. Barreca, The distribution of phthalate esters in indoor dust of Palermo (Italy), Environ. Geochem. Health 35 (2013) 613-624.

[4] G.W. Wolfe, K.A. Layton, Multigeneration reproduction toxicity study in rats diethylhexylphthalate: multigenerational reproductive assessment by continuous breeding when administered to Sprague-Daw ley rats in the diet, TRC Study no. 7244-200, The Immune Research Corporation, Gaithersburg, Mary-land, 2003.

[5] R.M. Maerteens, J. Bailey, P.A. White, The mutagenic hazards of settled house dust: a review, Mutat. Res. 567 (2004) 401-425.

[6] R. Kavlock, K. Boekelheide, R. Chapin, M. Cunningham, E. Faustman, P. Foster, Center for the evaluation of risks to human reproduction: phthalates expert panel report on the reproductive and developmental toxicity of butyl benzylphthalate, Reprod. Toxicol. 16 (2002) 453-653.

[7] T.K. Lau, W. Chu, N. Graham, The degradation of endocrine disruptor di-n-butyl phthalate by UV irradiation: a photolysis and product study, Chemosphere 60 (2005) 1045-1053.

[8] A. Pace, S. Barreca, Environmental organic photochemistry: advages and perspectives, Curr. Org. Chem. 14 (2013) 3032-3041. 
[9] D. Koniecki, R. Wang, R.P. Moody, J. Zhu, Phthalates in cosmetic and personal care products: concentrations and possible dermal exposure, Environ. Res. 111 (2011) 329-336.

[10] T. Schettler, Human exposure to phthalates via consumer products, Int. J. Androl. 29 (2006) 134-139.

[11] C. Perez-Feas, M.C. Barciela-Alonso, P. Bermejo-Barrera, Presence of phthalates in contact lens and cleaning solutions, Microchem. J. 99 (2001) 108-113.

[12] M. Bonini, E. Errani, G. Zerbinati, E. Ferri, S. Girotti, Extraction and gas chromatographic evaluation of plasticizers content in food packaging films, Microchemical 90 (2008) 9031-9036.

[13] S.Y. Yuan, C. Liu, C.S. Liao, Occurrence and microbial degradation of phthalate esters in Taiwan river sediments, Chemosphere 49 (2002) 1295-1299.

[14] Y. Sha, X. Xia, Z. Yang, G.H. Huang, Distribution of PAEs in the middle and lower reaches of the Yellow River, China, Environ. Monit. Assess. 124 (2007) 277-287.

[15] P. Wang, S.L. Wang, C.Q. Fan, Atmospheric distribution of particulate- and gas-phase phthalic esters (PAEs) in a metropolitan city, Nanjing, East China, Chemosphere 72 (2008) 1567-1572.

[16] F. Wang, X.H. Xia, Y.J. Sha, Distribution of phthalic acid esters in Wuhan section of the Yangtze River, China, Hazard. Mater. 154 (2008) 317-324.

[17] X. Xia, L. Yang, Q. Bu, R. Liu, Levels, distribution, and health risk of phthalate esters in urban soils of Beijing, China, J. Environ. Qual. 40 (2011) 1643-1651.

[18] C.J. Weschler, T. Salthammer, H. Fromme, Partitioning of phthalates among the gas phase, airborne particles and settled dust in indoor environment, Atmos. Environ. 42 (2008) 1449-1460.

[19] J.V. Cizdziel, V.F. Hodge, Attics as archives for house infiltrating pollutants: trace elements and pesticides in attic dust and soil from southern Nevada and Utah, Microchem. J. 64 (2000) 85-92.

[20] S. Barreca, S. Orecchio, A. Pace, Photochemical sample treatment for extracts clean up in PCB analysis from sediments, Talanta 103 (2013) 349-354.

[21] B. Berge, The Ecology of Building Materials, 2nd edition Elsevier, USA, 2009.

[22] B. Kolarik, C.G. Bornehag, K. Naydenov, J. Sundell, P. Stavova, O.F. Nielsen, The concentrations of phthalates in settled dust in Bulgarian homes in relation to building characteristic and cleaning habits in the family, Atmos. Environ. 42 (2008) 8553.

[23] M.R. Mannino, S. Orecchio, Polycyclic aromatic hydrocarbons (PAHs) in indoor dust matter of Palermo (Italy) area: extraction, GC-MS analysis, distribution and sources, Atmos. Environ. 42 (2008) 1801-1817.

[24] A. Gianguzza, L. Culotta, M.R. Mannino, S. Orecchio, Pelargonium leaves as bioaccumulator of Polynuclear Aromatic Hydrocarbons: analytical method and evaluation of sources and air quality in Palermo area, Fresenius Environ. Bull. 15 (2006) 928-935.

[25] S. Orecchio, PAHs associated with leaves of Quercus ilex L.: extraction, GC-MS analysis, distribution and sources. Assessment of air quality in the Palermo (Italy) area, Atmos. Environ. 41 (2007) 8669-8680.

[26] A. Gianguzza, L. Culotta, S. Orecchio, Absorption of polycyclic aromatic hydrocarbons by pinus bark: analytical method and use for environmental pollution monitoring in the Palermo area (Sicily, Italy), Environ. Res. 107 (2008) 371-379.

[27] S. Orecchio, D. Amorello, Platinum and rhodium associated with the leaves of Nerium oleander L; ; analytical method using voltammetry; assessment of air quality in the Palermo (Italy) area, J. Hazard. Mater. 174 (2010) 720-727.

[28] S. Orecchio, Contamination from polycyclic aromatic hydrocarbons (PAHs) in the soil of a botanical garden localized next to a former manufacturing gas plant in Palermo (Italy), J. Hazard. Mater. 180 (2010) 590-601.

[29] S. Orecchio, D. Amorello, Platinum levels in urban soils from Palermo (Italy); analytical method using voltammetry, Microchem. J. 99 (2011) 283-288.

[30] G. Hizal, Q.Q. Zhu, Ch.H. Fischer, P.M. Fritz, W. Schnabel, On the photolysis of phthalic acid dialkyl esters: a product analysis study, J. Photochem. Photobiol. A Chem. 72 (1993) 147-152.

[31] A.I. Balabanovich, W. Schnabel, On the photolysis of phthalic acid dimethyl and diethyl ester: a product analysis study, J. Photochem. Photobiol. A Chem. 113 (1998) 145-153.

[32] C.A. Staple, D.R. Peterson, T.F. Parkerton, W.J. Adams, The environmental fate of the phthalates esters: a literature review, Chemosphere 35 (1997) 667-749. 